\title{
Recuentos cromosómicos en plantas que crecen en Chile. IV
}

\section{Chromosomes report from plants that grown in Chile. IV}

\author{
Carlos M. Baeza ${ }^{1 *}$, Patricio Peñallillo ${ }^{2}$, Patricio Novoa ${ }^{3}$, Marcelo Rosas $^{4}$, Víctor L. Finot ${ }^{5} \&$ \\ EDUARDO RUIZ
}

'Facultad de Ciencias Naturales y Oceanográficas, Departamento de Botánica, Casilla 160-C, Universidad de Concepción, Concepción, Chile.

${ }^{2}$ Universidad de Talca, Instituto de Biología Vegetal y Biotecnología, Talca, Chile.

${ }^{3}$ Corporación Nacional Forestal, Jardín Botánico Nacional, Viña del Mar, Chile.

${ }^{4}$ Programa de Doctorado en Sistemática y Biodiversidad, Departamento de Botánica, Casilla 160-C, Universidad de Concepción, Concepción, Chile.

${ }^{5}$ Departamento de Producción Animal, Facultad de Agronomía, Universidad de Concepción, Chillán.

*cbaeza@udec.cl

\begin{abstract}
RESUMEN
Se entregan números cromosómicos de 19 especies de la flora vascular de Chile, obtenidos de raíces provenientes de semillas germinadas en placas Petri o de material recolectado en terreno creciendo en maceteros en invernadero. Nuevos registros citológicos para la flora de Chile son: Alstroemeria magnifica Herbert subsp. magenta (Bayer) M. Muñoz ( $2 \mathrm{n}$ $=16)$, Astragalus looseri I.M. Johnst. $(2 \mathrm{n}=22)$, Centaurea melitensis L. $(2 \mathrm{n}=24)$, Centaurea solstitialis L. $(2 \mathrm{n}=16)$, Erigeron andicola DC. $(2 \mathrm{n}=36)$, Montiopsis potentilloides (Barnéoud) D.I. Ford $(2 \mathrm{n}=20)$, Persea lingue (Ruiz et Pav.) Nees ex Kopp $(2 \mathrm{n}=24)$, Phycella cyrtanthoides (Sims) Lindl. $(2 \mathrm{n}=16)$, Picris echioides L. $(2 \mathrm{n}=10)$, Rhodophiala aff. maculata (L'Hér.) Ravenna $(2 \mathrm{n}=18)$, Teline monspessulana (L.) K. Koch $(2 \mathrm{n}=48)$ y Ulex europaeus L. subsp. europaeus $(2 \mathrm{n}=96)$.
\end{abstract}

Palabras clave: Números cromosómicos, flora vascular de Chile.

\begin{abstract}
Chromosome numbers of 19 Chilean species of vascular plants were studied using root-tip mitotic metaphases. New records for the chilean flora are: Alstroemeria magnifica Herbert subsp. magenta (Bayer) M. Muñoz ( $2 \mathrm{n}=16)$, Astragalus looseri I.M. Johnst. $(2 \mathrm{n}=22)$, Centaurea melitensis L. $(2 \mathrm{n}=24)$, Centaurea solstitialis L. $(2 \mathrm{n}=16)$, Erigeron andicola DC. $(2 \mathrm{n}=36)$, Montiopsis potentilloides (Barnéoud) D.I. Ford $(2 \mathrm{n}=20)$, Persea lingue (Ruiz et Pav.) Nees ex Kopp $(2 \mathrm{n}=24)$, Phycella cyrtanthoides (Sims) Lindl. $(2 \mathrm{n}=16)$, Picris echioides L. $(2 \mathrm{n}=10)$, Rhodophiala aff. maculata (L'Hér.) Ravenna $(2 \mathrm{n}=18)$, Teline monspessulana $(\mathrm{L}.) \mathrm{K}$. Koch $(2 \mathrm{n}=48)$, and Ulex europaeus L. subsp. europaeus $(2 \mathrm{n}=96)$.
\end{abstract}

KeYwords: Chromosome numbers, Chilean vascular flora.

\section{INTRODUCCIÓN}

Según Stuessy \& Taylor (1995) la flora chilena ha sido muy poco documentada en recuentos cromosómicos. Jara-Seguel \& Urrutia (2012) señalan que el número cromosómico es el carácter más estudiado en la citogenética de la flora chilena y se tiene información de alrededor de 160 taxones, incluidas especies, subespecies, variedades e híbridos naturales. También señalan que existen datos citogenéticos de un $2,8 \%$ de la flora de angiospermas chilenas correspondientes a 58 géneros y 34 familias. Tanto el recuento cromosómico como datos citogenéticos pueden constituir una valiosa información para complementar estudios en áreas como la sistemática y biodiversidad de un grupo determinado de vegetales. Datos de Marticorena (1990) indican que cerca del $45 \%$ de la flora chilena es endémica y un muy bajo porcentaje de ella es conocida citológicamente, lo que puede dificultar la interpretación de estudios sistemáticos, evolutivos y filogenéticos. En este trabajo se entregan números cromosómicos de 21 poblaciones de especies de plantas chilenas que complementan las ya publicadas en Baeza et al. 2001, 2004 y 2007. Aunque se reportan nuevas poblaciones de especies ya contadas anteriormente, sin variación en el número de ploidía, siempre es importante 
hacer conteos a nivel población considerando distintos lugares de colecta, porque muchas veces aparecen poliploides que enriquecen la información citotaxonómica del país.

\section{MATERIALES Y MÉTODO}

Se utilizó la metodología de Baeza et al. (2007) para realizar este estudio y que consistió en lo siguiente: puntas de raíces de 5-10 $\mathrm{mm}$ de longitud, obtenidas a partir de plantas cultivadas en invernadero o de semillas germinadas en placas petri, fueron pretratadas con una solución de 8-hidroxiquinolina $(2 \mathrm{mM})$ por $24 \mathrm{~h}$ a $4{ }^{\circ} \mathrm{C}$. Posteriormente se fijaron en etanol / ácido acético (3:1) por $24 \mathrm{~h}$ y se almacenaron a $5{ }^{\circ} \mathrm{C}$. Posterior a la fijación se realizó una hidrólisis ácida con $\mathrm{HCl} 0,5 \mathrm{~N}$ durante $18 \mathrm{~min}$ a $45^{\circ} \mathrm{C}$, luego se lavó con agua destilada en 2 oportunidades y por último se tiñó el ápice de la raíz con orceina acética al $1 \%$ y se realizó el aplastado. Se contaron como mínimo 5 individuos por cada población analizada. El conteo y observación de los cromosomas se realizó usando un microscopio Zeiss Axioskop con cámara de video monocromática incluida. Las microfotografías fueron analizadas y contrastadas con Paint Shop Pro X2.

\section{RESULTADOS}

La Tabla I resume la información de la familia, especie, localidad, número de colección, número de metafases observadas y la fotografía de los cromosomas de los taxones investigados. El asterisco $\left(^{*}\right)$ indica recuento nuevo para la flora chilena. Son nuevos conteos para la flora chilena Alstroemeria magnifica subsp. magenta, Astragalus looseri, Centaurea melitensis, Centaurea solstitialis, Erigeron andicola, Montiopsis potentilloides, Persea lingue, Phycella cyrtanthoides, Picris echioides, Rhodophiala aff. maculata, Teline monspessulana y Ulex europaeus subsp. europaeus. El resto de las especies reportadas en este trabajo ya habían sido contadas en otras oportunidades, pero se trata de nuevas localidades para el país.

Tabla I. Localidad y número de recolección, número de metafases, número diploide y figura de cada una de las poblaciones analizadas.

Table I. Location and collection number, number of metaphase, diploid number, and figure of the analyzed populations.

\begin{tabular}{|c|c|c|c|c|c|}
\hline FAMILIA & EsPECIE & Datos & $\begin{array}{c}\mathrm{N}^{\circ} \text { DE } \\
\text { METAFASES }\end{array}$ & $2 \mathrm{~N}$ & FIGURA \\
\hline \multirow[t]{2}{*}{ Alstroemeriaceae } & $\begin{array}{l}\text { Alstroemeria magnifica } \\
\text { Herbert subsp. magenta } \\
\text { (Bayer) M. Muñoz }\end{array}$ & $\begin{array}{l}\text { IV Región de Coquimbo, Prov. Choapa, Fundo Palo } \\
\text { Colorado, } 5 \mathrm{~km} \text { al norte de Puente Quilimarí, frente } \\
\text { al Cerro Tentén. } 80 \mathrm{~m}\left(32^{\circ} 05^{\prime} \text { S- } 71^{\circ} 30^{\prime} \mathrm{W}\right) .01-\mathrm{XI}- \\
\text { 2013. C. Baeza } 4381 \text { (CONC). }\end{array}$ & 25 & $16^{*}$ & $1 \mathrm{~A}$ \\
\hline & $\begin{array}{l}\text { Bomarea salsilla (L.) } \\
\text { Mirb. }\end{array}$ & $\begin{array}{l}\text { VIII Región del Biobío, Prov. Concepción, San Pe- } \\
\text { dro de La Paz. Villa El Recodo, km. 2,5, cerros ale- } \\
\text { daños a la Villa. } 45 \text { m ( } 36^{\circ} 51 \text { 'S-73º4'W). 31-XII- } \\
\text { 2011. C. Baeza } 4353 \text { A (CONC). }\end{array}$ & 15 & 18 & $1 \mathrm{~B}$ \\
\hline \multirow[t]{4}{*}{ Amaryllidaceae } & $\begin{array}{l}\text { Famatina cisandina } \\
\text { Ravenna }\end{array}$ & $\begin{array}{l}\text { VII Región del Maule, Prov. Curicó, Los Queñes. } \\
\text { Camino hacia Paso Vergara, pasado el desvío hacia } \\
\text { cemento Bío-Bío. } 1433 \mathrm{~m}\left(35^{\circ} 03 \text { 'S-70³0’W). 25- }\right. \\
\text { IV-2009. L. Letelier \& P. Peñailillo s.n. (Herbario } \\
\text { Universidad de Talca). }\end{array}$ & 18 & 18 & $1 \mathrm{C}$ \\
\hline & $\begin{array}{l}\text { Phycella cyrtanthoides } \\
\text { (Sims) Lindl }\end{array}$ & $\begin{array}{l}\text { V Región de Valparaíso, Prov. Valparaíso, Viña del } \\
\text { Mar. Jardín Botánico Nacional, ladera del cerro al } \\
\text { lado del vivero. } 30 \mathrm{~m}\left(33^{\circ} 23^{\prime} \mathrm{S}-71^{\circ} 30^{\prime} \mathrm{W}\right) .15-\mathrm{IX}- \\
\text { 2009. P.Novoa s.n. (JBN). }\end{array}$ & 15 & $16^{*}$ & $1 \mathrm{D}$ \\
\hline & $\begin{array}{l}\text { Rhodolirium laetum } \\
\text { (Phil.) Ravenna }\end{array}$ & $\begin{array}{l}\text { II Región de Antofagasta, Prov. Antofagasta, Tal- } \\
\text { tal, Quebrada de Miguel Díaz. } 276 \text { m (24³2’S- } \\
70^{\circ} 33^{\prime} \text { 'W). 29-X-2010. M. Rosas } 7041 \text { (Herbario } \\
\text { INIA, Vicuña, Chile). }\end{array}$ & 10 & 16 & $1 \mathrm{E}$ \\
\hline & $\begin{array}{l}\text { Rhodophiala advena } \\
\text { (Ker Gawl.) Traub }\end{array}$ & $\begin{array}{l}\text { Región Metropolitana, Prov. Santiago, Cuesta Lo } \\
\text { Prado, cima y cerca de las antenas. } 966 \text { m }\left(33^{\circ} 27^{\prime} \text { S- }\right. \\
70^{\circ} 57^{\prime} \text { W). 4-III-2005. M. Rosas \& R. Trincado } \\
2367 \text { (Herbario INIA). }\end{array}$ & 14 & 18 & $1 \mathrm{~F}$ \\
\hline
\end{tabular}




\begin{tabular}{|c|c|c|c|c|c|}
\hline FAMILIA & EsPECIE & DAtOS & $\begin{array}{c}\mathrm{N}^{\mathrm{o}} \mathrm{DE} \\
\text { METAFASES }\end{array}$ & $2 \mathrm{~N}$ & FIGURA \\
\hline & $\begin{array}{l}\text { Rhodophiala aff. } \\
\text { maculata (L'Hér.) } \\
\text { Ravenna }\end{array}$ & $\begin{array}{l}\text { VIII Región del Biobío, Prov. Concepción, Hual- } \\
\text { pén. Recinto Universidad de Concepción. } 5 \mathrm{~m} \\
\left(36^{\circ} 48^{\prime} \mathrm{S}-73^{\circ} 10^{\prime} \mathrm{W}\right) .20 \text {-XI-2011. C. Baeza } 4340 \\
\text { (CONC). }\end{array}$ & 20 & $18 *$ & $1 \mathrm{G}$ \\
\hline & & $\begin{array}{l}\text { Prov. Nuble. Cerca de Cobquecura, en la costa, } 3 \\
\text { m }\left(36^{\circ} 07 \mathrm{~S}-72^{\circ} 48^{\prime} \text { W). 18-XII-2010. C. Baeza } 4338\right. \\
(\text { CONC). }\end{array}$ & 16 & $18^{*}$ & \\
\hline \multirow[t]{8}{*}{ Asteraceae } & Centaurea melitensis L. & $\begin{array}{l}\text { Región Metropolitana, Prov. Santiago, Las Condes. } \\
\text { Quebrada San Ramón. } 895 \text { m (33²6’S-70³0’W.). } \\
\text { 10-I-2004. S. Gómez s.n. (CONC). }\end{array}$ & 15 & $24 *$ & $1 \mathrm{H}$ \\
\hline & Centaurea solstitialis L. & $\begin{array}{l}\text { Región Metropolitana, Prov. de Santiago, Maipú. } \\
\text { Quebrada de La Plata, } 740 \text { m ( } 33^{\circ} 29^{\prime} \text { S- } 70^{\circ} 54^{\prime} \text { W). } \\
\text { 4-I-2004. S. Gómez s.n. (CONC). }\end{array}$ & 14 & $16^{*}$ & $1 \mathrm{I}$ \\
\hline & Erigeron andicola DC. & $\begin{array}{l}\text { Región Metropolitana, Prov. de Santiago, Lo Bar- } \\
\text { nechea. Canchas de esquí sobre La Parva. } 2.800 \text { m } \\
\left(33^{\circ} 20^{\prime} S-70^{\circ} 17^{\prime} \mathrm{W}\right) .10-\mathrm{II}-2013 \text {. G. Valencia s.n. } \\
\text { (CONC). }\end{array}$ & 12 & $36^{*}$ & $1 \mathrm{~J}$ \\
\hline & $\begin{array}{l}\text { Haplopappus donianus } \\
\text { (Hook. et Arn.) Reiche }\end{array}$ & $\begin{array}{l}\text { V Región Valparaíso, Prov. Valparaíso. Quebra- } \\
\text { da La Fábrica, Playa Ancha. } 270 \text { m (3303’S- } \\
71^{\circ} 39^{\prime} \text { W). 11-XII- 2007. C. Baeza } 4269 \text { A (CONC). }\end{array}$ & 14 & 10 & $1 \mathrm{~K}$ \\
\hline & $\begin{array}{l}\text { Hypochaeris radicata } \\
\text { L. }\end{array}$ & $\begin{array}{l}\text { VIII Región del Biobío, Prov. Arauco. Entre Quidi- } \\
\text { co y Tirúa. } 45 \mathrm{~m}\left(38^{\circ} 11^{\prime} \mathrm{S}-73^{\circ} 26^{\prime} \mathrm{W}\right) \text {. 24-IV-2004. } \\
\text { Baeza } 4210 \text { (CONC). }\end{array}$ & 10 & 8 & $1 \mathrm{~L}$ \\
\hline & & $\begin{array}{l}\text { Prov. Concepción. Comuna de Lota. Laguna La } \\
\left.\text { Posada. } 25 \text { m ( } 36^{\circ} 56^{\prime} S-73^{\circ} 09^{\prime} W\right) \text {. 23-IV-2006. C. } \\
\text { Baeza } 4259 \text { (CONC). }\end{array}$ & 10 & 8 & $1 \mathrm{LL}$ \\
\hline & Picris echioides L. & $\begin{array}{l}\text { VIII Región de Biobío, Prov. Concepción, Concep- } \\
\text { ción. Barrio Universitario. Sector cancha de fútbol. } \\
10 \text { m (36 } 36^{\circ} \text { 'S-7303’W). 13-IV-2009. C. Baeza } \\
4296 \text { (CONC). }\end{array}$ & 18 & $\begin{array}{l}10^{*} \\
20 *\end{array}$ & $\begin{array}{l}1 \mathrm{M} \\
1 \mathrm{~N}\end{array}$ \\
\hline & $\begin{array}{l}\text { Taraxacum officinale } \\
\text { Weber }\end{array}$ & $\begin{array}{l}\text { Región Metropolitana, Prov. Santiago, Lo Bar- } \\
\text { nechea. Cima del Cerro Franciscano. } 3.585 \mathrm{~m} \\
\left(33^{\circ} 19^{\prime} \mathrm{S}-70^{\circ} 15^{\prime} \mathrm{W}\right) .10-\mathrm{II}-2013 \text {. G. Valencia s.n. } \\
(\mathrm{CONC})\end{array}$ & 12 & $\begin{array}{l}24 \\
32\end{array}$ & $\begin{array}{l}1 \mathrm{O} \\
1 \mathrm{P}\end{array}$ \\
\hline \multirow[t]{3}{*}{ Fabaceae } & $\begin{array}{l}\text { Astragalus looseri } \\
\text { I.M.Johnst. }\end{array}$ & $\begin{array}{l}\text { Región Metropolitana, Prov. de Santiago, Lo Bar- } \\
\text { nechea. Canchas de esquí sobre La Parva. } 2.800 \text { m } \\
\left(33^{\circ} 20^{\prime} S-70^{\circ} 17^{\prime} \mathrm{W}\right) .10-\mathrm{II}-2013 \text {. G. Valencia s.n. } \\
\text { (CONC). }\end{array}$ & 17 & $22 *$ & $1 \mathrm{Q}$ \\
\hline & $\begin{array}{l}\text { Teline monspessulana } \\
\text { (L.) K. Koch }\end{array}$ & $\begin{array}{l}\text { VIII Región de Biobío, Prov. Concepción, Coronel, } \\
\text { Camino a Santa Juana, sector El Llano, km 14,8. } 249 \\
\text { m ( } 36^{\circ} 58^{\prime} \text { S- } 73^{\circ} 02^{\prime} \text { W). 15-IV-2012. C. Baeza } 4361 \\
\text { (CONC). }\end{array}$ & 12 & $48^{*}$ & $1 \mathrm{R}$ \\
\hline & $\begin{array}{l}\text { Ulex europaeus L. } \\
\text { subsp. europaeus }\end{array}$ & $\begin{array}{l}\text { VIII Región de Biobío, Prov. Concepción. Hualpén, } \\
\text { Parque Pedro del Rio Zañartu, cerca de la desem- } \\
\text { bocadura. } 3 \mathrm{~m}\left(36^{\circ} 48^{\prime} \mathrm{S}-73^{\circ} 10^{\prime} \mathrm{W}\right) .15-\mathrm{III}-2011 . \text { C. } \\
\text { Baeza } 4345 \text { A (CONC). }\end{array}$ & 11 & $96^{*}$ & $1 \mathrm{~S}$ \\
\hline Lauraceae & $\begin{array}{l}\text { Persea lingue (Ruiz et } \\
\text { Pav.) Nees }\end{array}$ & $\begin{array}{l}\text { IX Región de la Araucanía, Prov. Malleco, Purén, } \\
\text { Monumento Natural Contulmo. } 74 \text { m (38º0’S- } \\
73^{\circ} 11^{\prime} \text { W). 23-XII-2009. C. Baeza } 4312 \text { A (CONC). }\end{array}$ & 16 & $24 *$ & $1 \mathrm{~T}$ \\
\hline Portulacaceae & $\begin{array}{l}\text { Montiopsis } \\
\text { potentilloides } \\
\text { (Barnéoud) D.I. Ford }\end{array}$ & $\begin{array}{l}\text { Región Metropolitana, Prov. Santiago, Lo Barne- } \\
\text { chea, Canchas de esquí sobre La Parva. } 2.800 \mathrm{~m} \\
\left(33^{\circ} 19^{\prime} \mathrm{S}-70^{\circ} 15^{\prime} \mathrm{W}\right) .10-\mathrm{II}-2013 . \text { G. Valencia s.n. } \\
\text { (CONC). }\end{array}$ & 15 & $20 *$ & $1 \mathrm{U}$ \\
\hline
\end{tabular}



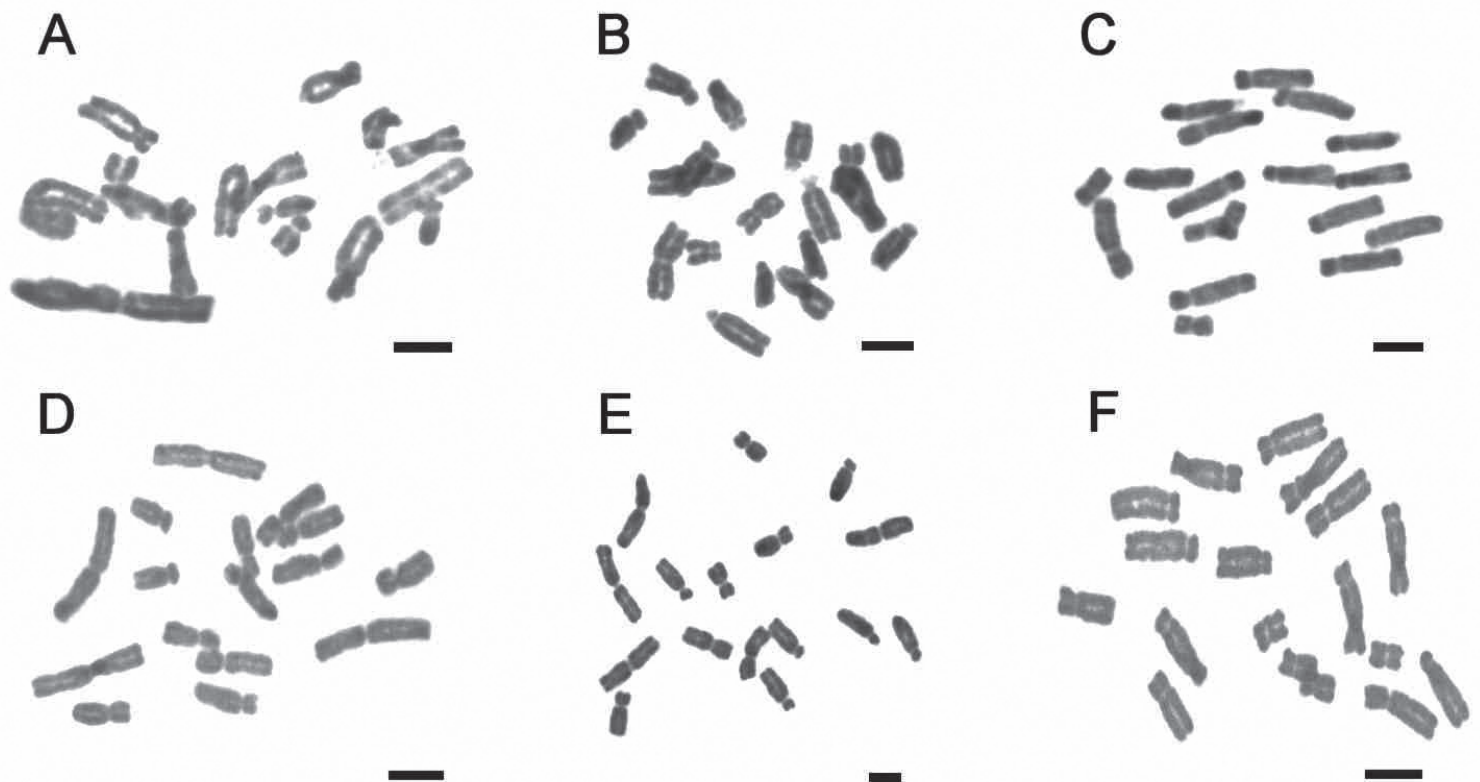

G

$\mathrm{H}$
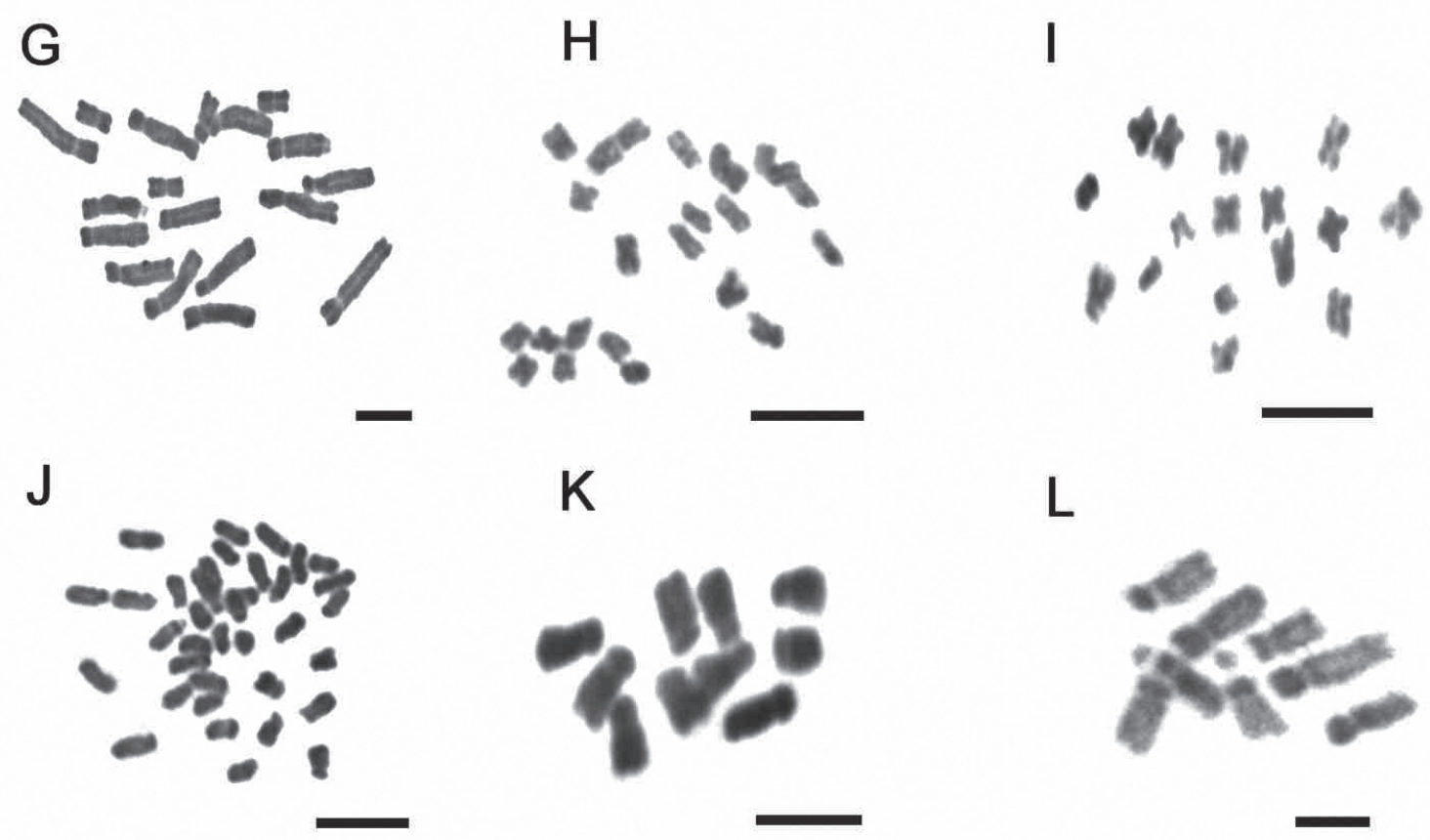

FIgURA 1. Placas metafásicas. A: Alstroemeria magnifica subsp. magenta $(2 \mathrm{n}=16)$. B: Bomarea salsilla $(2 \mathrm{n}=18)$. C: Famatina cisandina $(2 \mathrm{n}=18)$. D: Phycella cyrtanthoides $(2 \mathrm{n}=16)$. E: Rhodolirium laetum $(2 \mathrm{n}=16)$. F: Rhodophiala advena $(2 \mathrm{n}=18)$. G: Rhodophiala aff. maculata $(2 \mathrm{n}=18)$. H: Centaurea melitensis $(2 \mathrm{n}=24)$. I: Centaurea solstitialis $(2 \mathrm{n}=16)$. J: Erigeron andicola $(2 \mathrm{n}=36) . \mathrm{K}$ : Haplopappus donianus $(2 \mathrm{n}=10)$. L: Hypochaeris radicata $($ Baeza $4210,2 \mathrm{n}=8)$.

Figure 1. Metaphase plates. A: Alstroemeria magnifica subsp. magenta $(2 \mathrm{n}=16)$. B: Bomarea salsilla $(2 \mathrm{n}=18)$. C: Famatina cisandina $(2 \mathrm{n}=18)$. D: Phycella cyrtanthoides $(2 \mathrm{n}=16)$. E: Rhodolirium laetum $(2 \mathrm{n}=16)$. F: Rhodophiala advena $(2 \mathrm{n}=18)$. G: Rhodophiala aff. maculata $(2 \mathrm{n}=18)$. H: Centaurea melitensis $(2 \mathrm{n}=24)$. I: Centaurea solstitialis $(2 \mathrm{n}=16)$. J: Erigeron andicola $(2 \mathrm{n}=36)$. K: Haplopappus donianus $(2 \mathrm{n}=10)$. L: Hypochaeris radicata $($ Baeza $4210,2 \mathrm{n}=8)$. 
LL

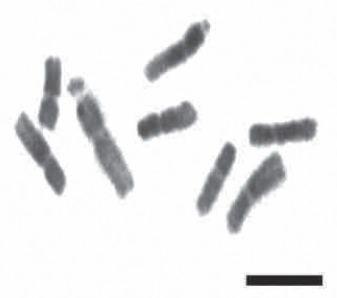

0
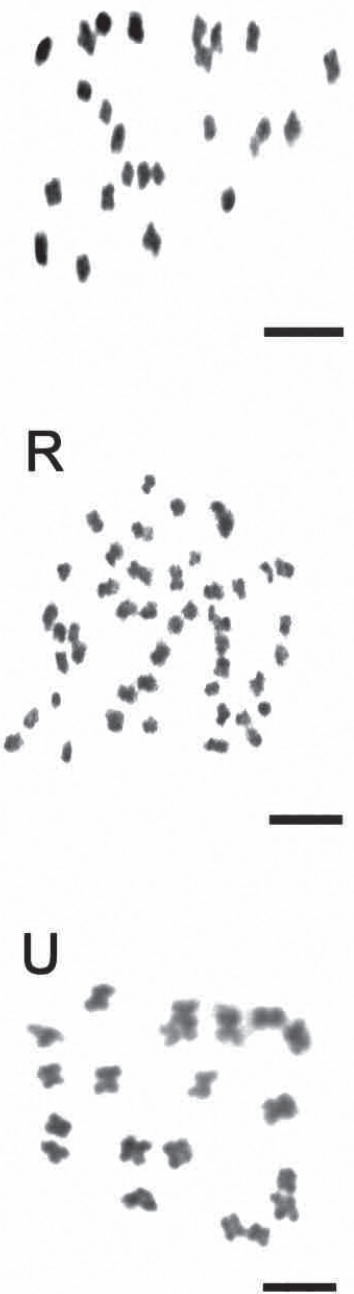

M

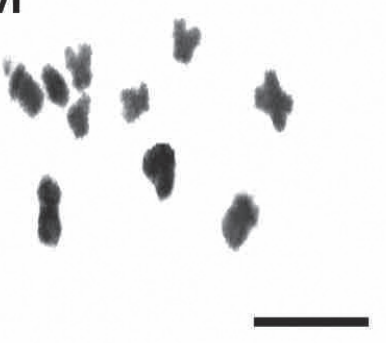

P

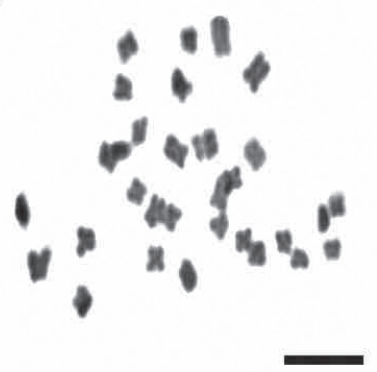

S

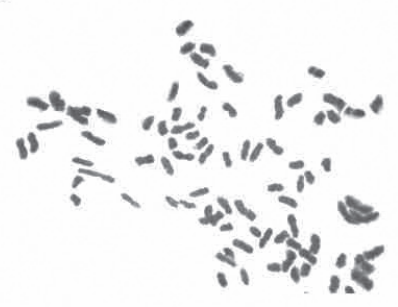

N

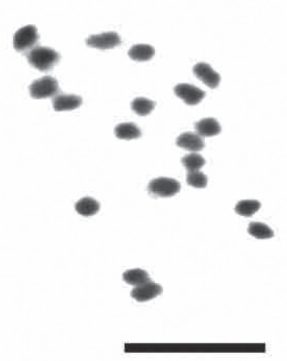

Q

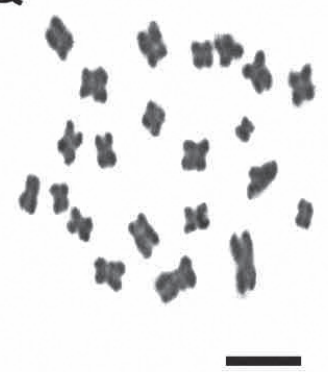

T

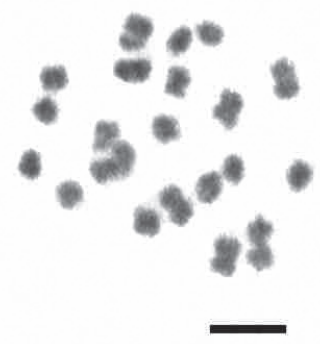

Continuación Figura 1. Placas metafásicas. LL: Hypochaeris radicata (Baeza 4259, $2 \mathrm{n}=8)$. M: Picris echioides $(2 \mathrm{n}=10)$. N: Picris echioides $(2 \mathrm{n}=4 \mathrm{x}=20)$. O: Taraxacum officinale $(3 \mathrm{n}=3 \mathrm{x}=24)$. P: Taraxacum officinale $(2 \mathrm{n}=4 \mathrm{x}=32)$. Q: Astragalus looseri $(2 \mathrm{n}=$ 22). R: Teline monspessulana $(2 \mathrm{n}=48)$. S: Ulex europaeus subsp. europaeus $(2 \mathrm{n}=6 \mathrm{x}=96)$. T: Persea lingue $(2 \mathrm{n}=24)$. U: Montiopsis potentilloides $(2 \mathrm{n}=20)$. La barra corresponde a $5 \mu \mathrm{m}$.

Continuation Figure 1. Metaphase plates. LL: Hypochaeris radicata (Baeza 4259, $2 \mathrm{n}=8)$. M: Picris echioides $(2 \mathrm{n}=10)$. N: Picris echioides $(2 \mathrm{n}=4 \mathrm{x}=20)$. O: Taraxacum officinale $(3 \mathrm{n}=3 \mathrm{x}=24)$. P: Taraxacum officinale $(2 \mathrm{n}=4 \mathrm{x}=32)$. Q: Astragalus looseri $(2 \mathrm{n}=$ 22). R: Teline monspessulana $(2 \mathrm{n}=48)$. S: Ulex europaeus subsp. europaeus $(2 \mathrm{n}=6 \mathrm{x}=96)$. T: Persea lingue ( $2 \mathrm{n}=24)$. U: Montiopsis potentilloides $(2 \mathrm{n}=20)$. Scale bar $5 \mu \mathrm{m}$. 


\section{DISCUSIÓN}

De los 21 conteos poblacionales que se presentan en este trabajo, 12 de ellos constituyen nuevos registros para la flora de Chile: Alstroemeria magnifica subsp. magenta $(2 \mathrm{n}$ $=16)$, Astragalus looseri $(2 \mathrm{n}=22)$, Centaurea melitensis $(2 \mathrm{n}=24)$, Centaurea solstitialis $(2 \mathrm{n}=16)$, Erigeron andicola $(2 \mathrm{n}=36)$, Montiopsis potentilloides $(2 \mathrm{n}=20)$, Persea lingue $(2 \mathrm{n}=24)$, Phycella cyrtanthoides $(2 \mathrm{n}=18)$, Picris echioides $(2 \mathrm{n}=10)$, Rhodophiala aff. maculata $(2 \mathrm{n}$ $=18)$, Teline monspessulana $(2 \mathrm{n}=48)$ y Ulex europaeus subsp. europaeus $(2 \mathrm{n}=96)$. A continuación se entregan antecedentes de los recuentos encontrados en este trabajo relacionados con la familia a la cual pertenece cada taxón. Se hace mención a conteos previos y en el caso de las especies malezoides analizadas se entrega información general respecto a su distribución en el país.

Alstroemeriaceae: existen recuentos cromosómicos previos para Alstroemeria magnifica subsp. magnifica de 2n = 16 (Buitendijk \& Ramanna 1996; Buitendijk et al. 1997, 1998; De Jeu et al. 1997), idéntico número encontrado en la subsp. magenta. Para Bomarea salsilla existía un registro de $2 n=18$ cromosomas para una población colectada en el cerro La Campana, en la Región de Valparaíso (PalmaRojas et al. 2007). En la actualidad, en la familia solo se han reportado especies con $2 \mathrm{n}=16$ y 18 .

Amaryllidaceae: existe un recuento previo de Famatina cisandina de $2 \mathrm{n}=18$ realizado por García et al. (2014). Este número cromosómico es el mismo encontrado en la población documentada en este trabajo. El recuento cromosómico para Phycella cyrtanthoides $(2 \mathrm{n}=16)$ es el primero para la flora chilena, existe un recuento de Phycella aff. cyrtanthoides realizado por García et al. (2014), esto significa, que no hay plena seguridad de la identidad de esa población. Existe un recuento cromosómico previo en Rhodolirium laetum (Garcia et al. 2014), idéntico al documentado en este trabajo $(2 \mathrm{n}=16)$. Rhodophiala advena ya había sido documentada en su número cromosómico por Baeza et al. (2006, como aff. advena) y por García et al. (2014). Esta nueva población también presenta $2 \mathrm{n}=18$ cromosomas. Rhodophiala aff. maculata $(2 \mathrm{n}=18)$ se documenta por primera vez para la flora de Chile. Esta población debe ser taxonómicamente estudiada, puesto que aún hay algunas dudas en la certeza de su determinación. Este es un problema bastante extendido en Rhodophiala, género de Amaryllidaceae ampliamente distribuido en Chile y que necesita de una urgente revisión taxonómica. En Chile sólo se conocen especies con $2 n=16$ y 18 dentro de la familia.

Asteraceae: son recuentos nuevos para la flora de Chile las dos especies de Centaurea analizadas citológicamente. Centaurea melitensis $(2 \mathrm{n}=24)$ es una planta europea introducida y frecuente en cultivos de trigo de secano y de lentejas, en suelos arcillosos (Matthei 1995). El mismo número cromosómico ha sido documentado para poblaciones de España, Grecia, Francia, Portugal, Argelia, Libia, Marruecos y Túnez (Bartolo et al. 1978; Hellwig 1994; Natarajan 1981; Natarajan 1988). Centaurea solstitialis (2n =16) también es una especie europea, tóxica, distribuida desde la Región de Valparaíso hasta la Región del Maule (Quiroz et al. 2009). Existe un recuento para Argentina ( $\mathrm{n}=$ 8, Turnet et al. 1979), y para países europeos como Albania, Bulgaria, Georgia, entre otros (Balstisberger 1993; Bancheva \& Greilhuber 2006; Guinochet 1957). Erigeron andicola (2n $=36$ ) es un nuevo recuento para la flora de Chile, solo existe un recuento previo de E. fernandezianus $(\mathrm{n}=27)$, material proveniente de la isla Robinson Crusoe del archipiélago de Juan Fernández (Sanders et al. 1983; Spooner et al. 1987). Existe un reporte cromosómico previo para Haplopappus donianus $(2 \mathrm{n}=10)$ de Brown \& Clark (1981), número idéntico encontrado en este trabajo. Hypochaeris radicata es una maleza común en Chile, ampliamente distribuida, y existen recuentos previos de poblaciones que crecen en el país (Baeza et al. 2001, 2004, 2007). Picris echioides es una especie anual, introducida, europea, de amplia distribución en Chile, también presente en Isla de Pascua (Matthei 1995). Este es el primer reporte cromosómico de esta especie para el país $(2 \mathrm{n}=10)$, encontrándose además individuos tetraploides $(2 n=4 x=20)$. Existen numerosos recuentos de esta especie en Europa, todos ellos $2 \mathrm{n}=10$ cromosomas (Keil \& Pinkava 1976; Natarajan 1981, 1988, Morton 1977; Pastor et al. 1990). Taraxacum officinale es una especie introducida euroasiática, muy distribuida en el país, invade áreas de cultivo, sitios perturbados, praderas, orillas de caminos (Quiroz et al 2009). Existe un recuento previo en Chile para esta especie, donde se encontró para tres poblaciones diferentes $2 \mathrm{n}=24$ (Molina-Montenegro et al 2013). En este trabajo se encontraron individuos $3 n=3 x=24$ y $2 \mathrm{n}=4 \mathrm{x}=32$; esta misma situación se ha documentado en otros países (Fazili et al 2011; Kashin et al 2003; Kliphuis \& Wieffering 1979; Verduijn 2004). La familia Asteraceae es la que presenta el mayor número de reportes cromosómicos en Chile, con números que van desde $2 \mathrm{n}=6$ a $2 \mathrm{n}=102$ (base de datos citológica CONC).

Fabaceae: el recuento cromosómico de Astragalus looseri $(2 \mathrm{n}=22)$ es nuevo para la flora chilena. El mismo número cromosómico tienen las siguientes especies chilenas: A. bellus (O.K.) R.E. Fries, A. curvicaulis (Clos) Reiche, A. darumbium (Bert. ex Savi) Clos, A. edmonstonei (Hook.) B.L. Rob., A. limariensis Muñoz y A. pehuenches Niederl. (Ledingham \& Pepper 1973). Teline monspessulana $(2 \mathrm{n}=48)$ es una especie introducida, europea, distribuida desde la Región de Valparaíso hasta la Región de Los Lagos, incluyendo el Archipiélago de Juan Fernández (Quiroz et al 2009). Es el primer recuento de esta especie para Chile. Similar número cromosómico ha sido reportado en Portugal (Fernandes \& Queiros 1978). Ulex europaeus subsp. europaeus $(2 \mathrm{n}=96)$ es una especie 
introducida en Chile, europea, muy nociva, considerada como una de las especies más invasoras de la flora chilena (Matthei 1995). Se reconocen dos subespecies: subsp. europaeus y subsp. latebracteatus (Mariz) Rothm. Se diferencian por la forma y tamaño de las bractéolas del cáliz y por el número cromosómico: la subsp. latebracteatus tiene $2 \mathrm{n}=64$ cromosomas y la subsp. europaeus $2 \mathrm{n}=96$ (Cubas 1999), por lo tanto, la subespecie que crece en Chile es la típica. Según la base de datos citológica CONC esta familia en Chile presenta, además, los siguientes números cromosómicos: $2 \mathrm{n}=12,14,16,18,20,24,28,32$ у 36 .

Lauraceae: Persea lingue $(2 \mathrm{n}=24)$, es una especie arbórea, endémica de Chile, cuyo reporte cromosómico es el primero para el país. Coincidentemente, Persea americana también presenta 2n $=24$ cromosomas (García 1975). Idéntico número cromosómico presenta otra Lauraceae chilena, Beilschmiedia (Baeza et al 2001).

Portulacaceae: el recuento para Montiopsis potentilloides es el primero para la flora chilena $(2 n=20)$. También se conoce el número cromosómico para Montiopsis umbellata $(2 \mathrm{n}=20)$, especie de los Andes de Chile central (Nyananyo 1986). Otros recuentos cromosómicos para esta familia en Chile son $2 \mathrm{n}=18$ y 54 (base de datos citológica CONC).

\section{AGRADECIMIENTOS}

Esta investigación fue financiada por el Proyecto Fondecyt $\mathrm{N}^{\circ}$ 1130349. Agradecemos también el apoyo prestado por la Universidad de Concepción, la Facultad de Ciencias Naturales y Oceanográficas y el Departamento de Botánica y también a Graciela Valencia y Susana Gómez por proveernos de material de algunas especies.

\section{BIBLIOGRAFÍA}

Baeza, C.M., G. Kottirsch, J. Espejo \& R. Reinoso. 2001. Recuentos cromosómicos en plantas chilenas. I. Gayana Botánica 58(2): 133-137.

Baeza, C., M. Vosyka \& T. Stuessy. 2004. Recuentos cromosómicos en plantas que crecen en Chile. II. Darwiniana 42(1-2): 2529.

BaEza, C., O. Schrader \& I. Escobar. 2006. Estudio del cariotipo en Rhodophiala aff. advena (Ker-Gawl.) Traub de la VIII Región de Chile. Kurtziana 32: 45-51.

Baeza, C., O. Schrader, A. Terrab, T. Stuessy, M. Rosas, E. Ruiz \& M. NegritTo. 2007. Recuentos cromosómicos en plantas que crecen en Chile III. Gayana Botánica 64(2): 175-183.

Bancheva, S. \& J. Greilhuber. 2006. Genome size in Bulgarian Centaurea s.l. (Asteraceae). Plant Systematics and Evolution 257: 95-117.

Balstisberger, M. 1993. Cytological investigations on Compositae from Albania. Candollea 48: 437-448.

Bartolo, G., S. Brullo \& P. Pavone. 1978. Numeri cromosomici per la flora Italiana: Informatore Botanico Italiano 10: 484-493.
Brown, G. \& W. Clark. 1981. Chromosome numbers in South American Haplopappus Cass. ( Compositae). American Journal of Botany 68: 1218-1221.

BuitendiJK, J. \& M. Ramanna 1996. Giemsa C-banded karyotypes of eight species of Alstroemeria L. and some of their hybrids. Annals of Botany 78: 449-457.

BuitendiJK, J., E. Boon \& M. Ramanna. 1997. Nuclear DNA content in twelve species of Alstroemeria L. and some of their hybrids. Annals of Botany 79: 343-353.

BuitendiJk, J., A. Peters, R. Jan-Quené \& M. Ramanna. 1998. Genome size variation and C-band polymorphism in Alstroemeria aurea, A. ligtu and A. magnifica (Alstroemeriaceae). Plant Systematics and Evolution 212: 87-106.

De Jeu, M., J. Lasschuit, A. Kuipers, S. Kamstra \& R. Visser. 1997. Characterisation and localization of repetitive DNA sequences in the ornamental Alstroemeria aurea Graham. Theoretical and Applied Genetic 94: 982-990.

Cubas, P. 1999. Ulex. En S. Castroviejo. Flora Ibérica VII (1): 212239.

Fazili, K., Y. Ali, S. Hussain, A. Andrab \& B. Wafai. 2011. Karyotype of apomictic Dandelion (Taraxacum officinale), a wild plant with high medicinal value. Recent Research in Science and Technology 3(10): 118-121.

Fernandes, A. \& M. QueIros. 1978. Contribution à la connaissance cytotaxinomique des Spermatophyta du Portugal. IV. Leguminosee (Suppl. 3). Boletim da Sociedade Broteriana 52: 79-164.

Garcia, A. 1975. Cytogenetic studies in the genus Persea (Lauraceae). I: Karyology of seven species. Canadian Journal of Genetic and Cytology 17: 173-180.

García, N., A. Meerow, D. Soltis \& P. Soltis. 2014. Testing Deep Reticulate Evolution in Amaryllidaceae Tribe Hippeastreae (Asparagales) with ITS and Chloroplast Sequence Data. Systematic Botany 39(1): 75-89.

Guinochet, M. 1957. Contribution a l'étude caryologique du genre Centaurea L. sens. lat. Bulletin de la Societe d'Histoire Naturelle de L' Afrique du Nord 48: 282-300.

Hellwig, F. 1994. Chromosomenzahlen aus der Tribus Cardueae (Compositae). Willdenowia 24: 219-248.

Jara-Seguel, P. \& J. Urrutia. 2012. Cytogenetics of Chilean angiosperms: Advances and prospects. Revista Chilena de Historia Natural 85: 1-12.

Kashin, A., A. Demotshco \& V. Martinova. 2003. Caryotype variation in population of apomictic and sexual species of agamic complexes of Asteraceae. Botaniceskij Žurnal 88(9): 35-51.

KeIL, D. \& D. PinKava. 1976. Chromosome counts and taxonomic notes for Compositae from the United States and Mexico. American Journal of Botany 63: 1393-1403.

KLIPHUIS, E. \& J. WiefFERING. 1979. In IOPB chromosome number reports LXIV. Taxon 28: 398-400.

Ledingham, G. \& B. Pepper. 1973. Chromosome numbers of some South American species of Astragalus. Kurtziana 7: 27-37.

Marticorena, C. 1990. Contribución a la estadística de la flora vascular de Chile. Gayana Botánica 47: 85-113.

Matrhei, O. 1995. Manual de las Malezas que Crecen en Chile. Santiago. 545 pp.

Molina-Montenegro, M., C. Palma-Rojas, Y. AlcayagaOlivares, R. Oses, L. Corcuera, L. Cavieres \& E. Gianoli. 
2013. Ecophysiological plasticity and local differentiation help explain the invasion success of Taraxacum officinale (dandelion) in South America. Ecography 36: 718-730.

Morton, J. 1977. A cytological study of the Compositae (excluding Hieracium and Taraxacum) of the British Isles. Watsonia 11: 211-223.

Natarajan, G. 1981. Chromosome number reports LXXII. Taxon 30: 698-699.

Natarajan, G. 1988. Etude caryosystématique de quelques dicotyledones de la Garrigue Languedocienne. Naturalia Monspeliensia 52: 85-123.

Nyananyo, B. 1986. Chromosome Number Reports 93. Taxon 35: 902-903.

Palma-Rojas, C., P. Jara-Seguel \& E. Von Brand. 2007. Karyological studies in Chilean species of Bomarea and Leontochir (Alstroemeriaceae). New Zealand Journal of Botany 45: 299-303.

Pastor, J., J. Diosdado, C. Bárbara, J. Vique \& E. Pérez. 1990. Números cromosómicos para la flora Española. 556-591. Lagascalia 15: 269-282.
Quiroz, C., A. Pauchard, A. Marticorena \& L. Cavieres. 2009. Manual de Plantas Invasoras del Centro-Sur de Chile. Laboratorio de Invasiones Biológicas. Concepción. 45 pp.

SAnders, R., T. Stuessy \& R. Rodríguez. 1983. Chromosome numbers from the flora of the Juan Fernandez Islands. American Journal of Botany 70: 799-810.

Spooner, D., T. Stuessy, D. Crawford \& M. Silva. 1987. Chromosome numbers from the flora of the Juan Fernandez Islands. II. Rhodora 89: 351-356.

Stuessy, T. \& C. TAYlor. 1995. Evolución de la flora chilena. En: C. Marticorena \& R. Rodríguez (eds.), Flora de Chile 1: 85-118. Ediciones Universidad de Concepción, Chile.

Turner, B., J. Bacon, L. Urbatsh \& B. Simpson. 1979. Chromosome numbers in South American Compositae. American Journal of Botany 66: 173-178.

Verduisn, M. 2004. Distribution, phenology and demography of sympatric sexual and asexual dandelions (Taraxacum officinale s.l.): geographic parthenogenesis on a small scale. Botanical Journal of the Linnean Society 82: 205218.

Recibido: 24.03 .16

Aceptado: 25.08.16 